\title{
Numerical modelling of the internal reflection and Goos-Hänchen shift of electromagnetic beam wave
}

\author{
L’ubomír Šumichrast, Jaroslav Franek, \\ Rastislav Dosoudil, Jozefa Červeňová*
}

\footnotetext{
Total internal reflection of plane waves is a well-known phenomenon. Some new aspects of the numerical treatment of the total internal reflection phenomena, concerning the beam-wave, are discussed.

K e y w o r d s: Goos-Hänchen shift, wave reflection, numerical treatment
}

\section{Introduction}

Reflection and transmission of a homogeneous plane wave at the plane boundary of two dielectric media is a well known phenomenon commonly treated in nearly all standard textbooks. Investigation of the internal reflection of inhomogeneous plane waves and spatially confined beam waves is subject of permanent interest since the discovery of the Goos-Hänchen shift [1]. There is a huge number of papers published over the last seventy years. concerning this topic, just a small non-representative sample is for illustration exemplified by $[2-6]$. Here we investigate first the effects occurring due to the total internal reflection of an inhomogeneous plane wave - the classical Goos-Hänchen shift and subsequently we consider spatially cofined beam wave. Special attention is paid to the numerical treatment of these effects by a simple approach using Fourier series.

\section{Propagation of a monochromatic plane} wave along the planar dielectric boundary

Let us consider the planar boundary $x=0$ between the two half-spaces, $x<0$ and $x>0$, filled respectively with the dielectric media having permittivities $\varepsilon_{1}$ and $\varepsilon_{2}$ accordingly to Fig. 1.

Consider the infinite non-homogeneous harmonic plane wave of angular frequency $\omega_{0}$, with the electric intensity and the magnetic intensity vectors $\mathbf{E}(\boldsymbol{r}, t)=\operatorname{Re}\{\widehat{\mathbf{E}}(\boldsymbol{r}, t)\}$ and $\mathbf{H}(\boldsymbol{r}, t)=\operatorname{Re}\{\widehat{\mathbf{H}}(\boldsymbol{r}, t)\}$ in complex representation of the form

$$
\begin{aligned}
\widehat{\mathbf{E}}(\boldsymbol{r}, t) & =\widehat{\mathbf{E}}(\boldsymbol{r}) \exp \left(j \omega_{0} t\right), \\
\widehat{\mathbf{H}}(\boldsymbol{r}, t) & =\widehat{\mathbf{H}}(\boldsymbol{r}) \exp \left(j \omega_{0} t\right),
\end{aligned}
$$

propagating in direction $z$ with the wavenumber $k_{0}$, ie

$$
\begin{aligned}
\widehat{\mathbf{E}}(\boldsymbol{r}, t) & =\widehat{\mathbf{E}}(x, y) \exp \left(-j k_{0} z\right), \\
\widehat{\mathbf{H}}(\boldsymbol{r}, t) & =\widehat{\mathbf{H}}(x, y) \exp \left(-j k_{0} z\right) .
\end{aligned}
$$

In what follows, we shall consider only the two dimensional case, ie the wave transversal profile independent of $y$

$$
\widehat{\mathbf{E}}(x, y)=\widehat{\mathbf{E}}(x), \quad \widehat{\mathbf{H}}(x, y)=\widehat{\mathbf{H}}(x) .
$$

The mean value of the power flow density $\langle\mathbf{P}\rangle$ is given by the Poynting vector in the form

$$
\langle\mathbf{P}\rangle=\frac{1}{2} \operatorname{Re}\left\{\widehat{\mathbf{E}} \times \widehat{\mathbf{H}}^{*}\right\}
$$

The two-dimensional wave propagation may be formulated either in the transversal electric (TE) configuration with the electric and magnetic intensity vectors of the form

$$
\widehat{\mathbf{E}}(x)=E_{y}(x) \mathbf{u}_{y}, \quad \widehat{\mathbf{H}}(x)=H_{x}(x) \mathbf{u}_{x}+H_{z}(z) \mathbf{u}_{z} .
$$

or in the transversal magnetic (TM) configuration with $\widehat{\mathbf{E}}$ and $\widehat{\mathbf{H}}$ of the form

$$
\widehat{\mathbf{H}}(x)=H_{y}(x) \mathbf{u}_{y}, \widehat{\mathbf{E}}(x)=E_{x}(x) \mathbf{u}_{x}+E_{z}(x) \mathbf{u}_{z}
$$

For the TE configuration $E_{y}(x)$ fulfils in the respective half-spaces $x<0$ and $x>0$ the wave equations

$$
\begin{aligned}
& \frac{\partial^{2} E_{y}(x)}{\partial x^{2}}+\left(\beta_{1}^{2}-k_{0}^{2}\right) E_{y}(x)=0, x<0, \\
& \frac{\partial^{2} E_{y}(x)}{\partial x^{2}}+\left(\beta_{2}^{2}-k_{0}^{2}\right) E_{y}(x)=0, x>0,
\end{aligned}
$$

\footnotetext{
* Institute of Electrical Engineering, Slovak University of Technology in Bratislava, Slovakia, lubomir.sumichrast@stuba.sk
} 
where $\beta_{1}=\omega_{0} \sqrt{\mu \varepsilon_{1}}$ and $\beta_{2}=\omega_{0} \sqrt{\mu \varepsilon_{2}}$ are the phase constants in the respective media. The $\widehat{\mathbf{H}}(x)$ vector is determined using the Maxwell's equation

$$
\widehat{\mathbf{H}}(x)=\frac{j}{\omega_{0} \mu}\left[j k_{0} E_{y} \mathbf{u}_{x}+\frac{\partial E_{y}}{\partial x} \boldsymbol{u}_{z}\right] .
$$

For the TM configuration the wave equations for $H_{y}(x)$ are of the same form as (6) and (7) and the Maxwell's equation for determining $\widehat{\mathbf{E}}(x)$ reads

$$
\widehat{\mathbf{E}}(x)=-\frac{j}{\omega_{0} \varepsilon}\left[j k_{0} H_{y} \mathbf{u}_{x}+\frac{\partial H_{y}}{\partial x} \mathbf{u}_{z}\right]
$$

where $\varepsilon$ equals either $\varepsilon_{1}$ or $\varepsilon_{2}$ accordingly the respective region. For the sake of brevity we shall not pursue the TM case further.

\section{Non-homogeneous plane wave with cosine-like profile: $k_{0}<\min \left[\beta_{1}, \beta_{2}\right]$}

If the condition $k_{0}<\min \left[\beta_{1}, \beta_{2}\right]$ is fulfilled, $i e$ the wavenumber $k_{0}$ is smaller than any of the two phase constants $\beta_{1}, \beta_{2}$, then the general solution of (6) and (7) for TE configuration in respective domains $x<0$ and $x>0$ is of the type

$$
\begin{aligned}
& E_{y 1}(x)=A \exp \left(-j q_{1} x\right)+B \exp \left(j q_{1} x\right), \\
& E_{y 2}(x)=C \exp \left(j q_{2} x\right)+D \exp \left(-j q_{2} x\right),
\end{aligned}
$$

where $q_{1,2}=\sqrt{\beta_{1,2}^{2}-k_{0}^{2}}$. Taking into account continuity of the components $E_{y}, H_{x}$ and $H_{z}$ on the boundary $x=0$, one obtains

$$
\begin{aligned}
E_{y 1}(x)=E_{01}[ & \left.\exp \left(-j q_{1} x\right)+\rho \exp \left(j q_{1} x\right)\right]+ \\
& (1-\rho) E_{02} \exp \left(j q_{1} x\right), x<0,
\end{aligned}
$$

$$
\begin{aligned}
& E_{y 2}(x)=E_{02}\left[\exp \left(j q_{2} x\right)-\rho \exp \left(-j q_{2} x\right)\right]+ \\
&(1+\rho) E_{01} \exp \left(-j q_{2} x\right), x>0,
\end{aligned}
$$

where

$$
\rho=\left(q_{1}-q_{2}\right) /\left(q_{1}+q_{2}\right)
$$

is the reflection factor and $E_{01}, E_{02}$ are two arbitrary constants expressing the power flow density of the wave.

In fact (12) and (13) represent an interference of the two independent waves with amplitudes $E_{01}$ and $E_{02}$, each of them being reflected and refracted on the boundary $x=0$.

Using (12) and (13) the $\widehat{\mathbf{H}}(x, z)$ vector can be easily obtained from (8).

If both independent waves are of the same magnitude $E_{01}=E_{02}=E_{0}$ one obtains purely cosine-like amplitude profile of the non-homogeneous plane wave in the TE configuration propagating along the boundary

$$
\widehat{\mathbf{E}}_{1,2}(x)=\mathbf{u}_{y} 2 E_{0} \cos \left(q_{1,2} x\right),
$$

$$
\widehat{\mathbf{H}}_{1,2}(x)=\frac{-2 E_{0}}{\omega_{0} \mu}\left[\mathbf{u}_{x} k_{0} \cos \left(q_{1,2} x\right)+\boldsymbol{u}_{z} j q_{1,2} \sin \left(q_{1,2} x\right)\right],
$$

with the Poynting vector equal to

$$
\left\langle\mathbf{P}_{1,2}(x)\right\rangle=\mathbf{u}_{z} \frac{2 E_{0}^{2}}{\omega_{0} \mu} k_{0} \cos ^{2}\left(q_{1,2} x\right),
$$

ie resulting in no power transferred through the boundary plane $x=0$.

If only one independent wave exists, ie $E_{01}=E_{0}$, $E_{02}=0$, then in the halfspace $x<0$ it consists of the incident and reflected wave, with reflection coefficient $\rho$, and with resulting $\widehat{\mathbf{E}}, \widehat{\mathbf{H}}$ vectors equal to

$$
\widehat{\mathbf{E}}_{1}(x)=\mathbf{u}_{y} E_{0}\left\{\exp \left(-j q_{1} x\right)+\rho \exp \left(j q_{1} x\right)\right\},
$$

$$
\begin{aligned}
\widehat{\mathbf{H}}_{1}(x)=-\frac{E_{0}}{\omega_{0} \mu}\left\{\left[\boldsymbol{u}_{x} k_{0}-\right.\right. & \left.\boldsymbol{u}_{z} q_{1}\right] \exp \left(-j q_{1} x\right)+ \\
& \left.\rho\left[\mathbf{u}_{x} k_{0}+\mathbf{u}_{z} q_{1}\right] \exp \left(j q_{1} x\right)\right\} .
\end{aligned}
$$

The Poynting vector in the half space $x<0$ equals

$$
\begin{aligned}
\left\langle\mathbf{P}_{1}(x)\right\rangle=\frac{E_{0}^{2}}{2 \omega_{0} \mu}\left\{\mathbf { u } _ { z } k _ { 0 } \left[1+\rho^{2}+\right.\right. & \left.2 \rho \cos \left(2 q_{1} x\right)\right] \\
& \left.+\mathbf{u}_{x} q_{1}\left(1-\rho^{2}\right)\right\} .
\end{aligned}
$$

In the half space $x>0$ the resulting $\widehat{\mathbf{E}}, \widehat{\mathbf{H}}$ vectors are

$$
\begin{gathered}
\widehat{\mathbf{E}}_{2}(x)=\boldsymbol{u}_{y} \tau E_{0} \exp \left(-j q_{2} x\right) \\
\widehat{\mathbf{H}}_{2}(x)=-\frac{\tau E_{0}}{\omega_{0} \mu}\left[\boldsymbol{u}_{x} k_{0}-\boldsymbol{u}_{z} q_{2}\right] \exp \left(-j q_{2} x\right),
\end{gathered}
$$

with the Poynting vector equal to

$$
\left\langle\mathbf{P}_{2}(x)\right\rangle=\frac{\tau^{2} E_{0}^{2}}{2 \omega_{0} \mu}\left[\mathbf{u}_{z} k_{0}+\mathbf{u}_{x} q_{02}\right]
$$

where

$$
\tau=1+\rho=2 q_{1} /\left(q_{1}+q_{2}\right)
$$

is the transmission factor

The $x$-components $\left\langle P_{1 x}\right\rangle$ and $\left\langle P_{2 x}\right\rangle$ of the Poynting vectors $\left\langle P_{1}(x)\right\rangle$ and $\left\langle P_{2}(x)\right\rangle$ give the steady flow of the wave power density in direction perpendicular to the boundary and on the boundary $x=0$ are the same in medium $\varepsilon_{1}$ and $\varepsilon_{2}$, ie $\left\langle P_{1 x}\right\rangle=\left.\left\langle P_{2 x}\right\rangle\right|_{x=0}$, since $(1-$ $\left.\rho^{2}\right) q_{1}=\tau^{2} q_{2}$.

The $z$-components $\left\langle P_{1 z}\right\rangle$ and $\left\langle P_{2 z}\right\rangle$ give the steady power flow of the wave along the boundary $x=0$. The $\cos \left(2 q_{1} x\right)$ dependence of $\left\langle P_{1 z}\right\rangle$ in (20) is in fact due to the interference pattern of the incident and the reflected wave. On the boundary itself $\left\langle P_{1 z}\right\rangle$ and $\left\langle P_{2 z}\right\rangle$ are continuous, ie $\left\langle P_{1 z}\right\rangle=\left.\left\langle P_{2 z}\right\rangle\right|_{x=0}$, since $\left[1+\rho^{2}+\right.$ $\left.2 \rho \cos \left(2 q_{1} x\right)\right]\left.\right|_{x=0}=\tau^{2}$. 
4 Evanescent plane wave: $\beta_{1}>k_{0}>\beta_{2}$

Assuming $\varepsilon_{1}>\varepsilon_{2}$ and $\beta_{1}>k_{0}>\beta_{2}$ the solution of (6) and (7) for TE configuration reads

$$
\begin{aligned}
& \widehat{\mathbf{E}}_{1}(x)=\mathbf{u}_{y} E_{0}[\left.\exp \left(-j q_{0} x\right)+\rho \exp \left(j q_{0} x\right)\right]= \\
& \mathbf{u}_{y} 2 E_{0} \exp (j \Psi) \cos \left(q_{0} x+\Psi\right),
\end{aligned}
$$

$$
\begin{aligned}
\widehat{\mathbf{E}}_{2}(x)=\mathbf{u}_{y} \tau E_{0} \exp (-\varkappa x)= \\
\mathbf{u}_{y} 2 E_{0} \exp (j \Psi) \cos (\Psi) \exp (-\varkappa x),
\end{aligned}
$$

where

$$
\begin{aligned}
& q_{0}=q_{1}=\sqrt{\beta_{1}^{2}-k_{0}^{2}}, \varkappa=j q_{2}=\sqrt{k_{0}^{2}-\beta_{2}^{2}}, \\
& \rho=\exp (j 2 \Psi), \tau=2 \cos \Psi \exp (j \Psi), \tan \Psi=\varkappa / q_{0} .
\end{aligned}
$$

Here we have simply denoted $E_{01}=E_{0}$ since the second independent constant $E_{02}$ has to be set to zero from physical reasons - it leads to the $\exp (\varkappa x)$ dependence divergent for $x \rightarrow \infty$.

The pertaining $\widehat{\mathbf{H}}$ vectors are

$$
\begin{aligned}
\widehat{\mathbf{H}}_{1}(x)= & \frac{-2 E_{0}}{\omega_{0} \mu} \exp (j \Psi) \times \\
& {\left[\boldsymbol{u}_{x} k_{0} \cos \left(q_{0} x+\Psi\right)+\boldsymbol{u}_{z} j q_{0} \sin \left(q_{0} x+\Psi\right)\right], \quad(28) } \\
\widehat{\mathbf{H}}_{2}(x)= & \frac{-2 E_{0}}{\omega_{0} \mu} \exp (j \Psi) \cos (\Psi)\left[\boldsymbol{u}_{x} k_{0}-\mathbf{u}_{z} j \varkappa\right] \exp (-\varkappa x) .
\end{aligned}
$$

The power flow density in the respective medium equals

$$
\begin{aligned}
\left\langle\mathbf{P}_{1}(x)\right\rangle & =\boldsymbol{u}_{z} \frac{2 E_{0}^{2}}{\omega_{0} \mu} k_{0} \cos ^{2}\left(q_{0} x+\Psi\right), \\
\left\langle\mathbf{P}_{2}(x)\right\rangle & =\boldsymbol{u}_{z} \frac{2 E_{0}^{2}}{\omega_{0} \mu} k_{0} \cos ^{2}(\Psi) \exp (-2 \varkappa x) .
\end{aligned}
$$

Observe that in this case again, no power density flows through the boundary plane.

Thus, one arrives to the non-homogeneous plane wave with cosine-like profile in the medium $\varepsilon_{1}, x<0$, and exponentially evanescent profile in medium $\varepsilon_{2}, x>0$, propagating in the $z$-axis direction with the phase velocity $v_{f}=\omega_{0} / k_{0}$, in the medium $\varepsilon_{1}$ higher than the respective velocity of light $c_{1}=\omega_{0} / \beta_{1}$, and in the medium $\varepsilon_{2}$ smaller than the respective velocity of light $c_{2}=\omega_{0} / \beta_{2}$. Therefore this kind of wave is sometimes termed a "slow wave".

For the limiting case $k_{0}=\beta_{2}$ holds $\varkappa=0, \Psi=0$, $q_{0}=\sqrt{\beta_{1}^{2}-\beta_{2}^{2}}$. This leads either from (18) - (23) or from $(25)-(31)$ to

$$
\widehat{\mathbf{E}}_{1}(x)=\mathbf{u}_{y} 2 E_{0} \cos \left(q_{0} x\right), \quad \widehat{\mathbf{E}}_{2}(x)=\mathbf{u}_{y} 2 E_{0},
$$

$$
\begin{gathered}
\widehat{\mathbf{H}}_{1}(x)=\frac{-2 E_{0}}{\omega_{0} \mu}\left[\mathbf{u}_{x} \beta_{2} \cos \left(q_{0} x\right)+\mathbf{u}_{z} j q_{0} \sin \left(q_{0} x\right)\right] \\
\widehat{\mathbf{H}}_{2}(x)=\frac{-2 \beta_{2} E_{0}}{\omega_{0} \mu} \mathbf{u}_{x} \\
\left\langle\mathbf{P}_{1}(x)\right\rangle=\mathbf{u}_{z} \frac{2 E_{0}^{2}}{\omega_{0} \mu} \beta_{2} \cos ^{2}\left(q_{0} x\right) \\
\left\langle\mathbf{P}_{2}(x)\right\rangle=\mathbf{u}_{z} \frac{2 E_{0}^{2}}{\omega_{0} \mu} \beta_{2}
\end{gathered}
$$

$i e$ in the second medium $\varepsilon_{2}$ - the halfspace $x>0$ - a homogeneous plane wave of constant magnitude propagates in direction of the boundary without any exponentially attenuating tail.

\section{Standard approach to the plane wave reflection and transmission on the plane boundary}

Within the framework of the standard treatment of wave reflection and refraction the results presented in (18) and (21) represent, as depicted in Fig. 1, the electric intensity vectors of the incident, reflected and refracted homogeneous TE-polarised plane wave, expressed as

$$
\begin{aligned}
\widehat{\mathbf{E}}_{d}(\boldsymbol{r}) & =\mathbf{u}_{y} E_{0} \exp \left(-j \beta_{1} \mathbf{n}_{d} \cdot \mathbf{r}\right), \\
\widehat{\mathbf{E}}_{r}(\boldsymbol{r}) & =\rho \mathbf{u}_{y} E_{0} \exp \left(-j \beta_{1} \mathbf{n}_{r} \cdot \boldsymbol{r}\right), \\
\widehat{\mathbf{E}}_{p}(\boldsymbol{r}) & =\tau \mathbf{u}_{y} E_{0} \exp \left(-j \beta_{2} \boldsymbol{n}_{p} \cdot \boldsymbol{r}\right) .
\end{aligned}
$$

The propagation directions of the incident, reflected and transmitted plane waves are given by the unit vectors $\mathbf{n}_{d}$, $\mathbf{n}_{r}, \mathbf{n}_{p}$, where

$$
\begin{aligned}
& \beta_{1} \mathbf{n}_{d} \cdot \boldsymbol{r}=q_{1} x+k_{0} z, \\
& \beta_{1} \mathbf{n}_{r} \cdot \mathbf{r}=-q_{1} x+k_{0} z, \\
& \beta_{2} \mathbf{n}_{p} \cdot \boldsymbol{r}=q_{2} x+k_{0} z
\end{aligned}
$$

The incidence, reflection and refraction angles $\theta_{d}, \theta_{r}$, $\theta_{p}$ in Fig. 1 are defined by the directions of propagation

$$
\begin{aligned}
& \mathbf{n}_{d}=\cos \theta_{d} \boldsymbol{u}_{x}+\sin \theta_{d} \boldsymbol{u}_{z}, \\
& \mathbf{n}_{r}=-\cos \theta_{r} \mathbf{u}_{x}+\sin \theta_{r} \mathbf{u}_{z}, \\
& \mathbf{n}_{p}=\cos \theta_{p} \mathbf{u}_{x}+\sin \theta_{p} \boldsymbol{u}_{z} .
\end{aligned}
$$

Comparison with (18) and (21) yields

$$
\begin{aligned}
& q_{1}=\beta_{1} \cos \theta_{d}=\beta_{1} \cos \theta_{r}, \quad q_{2}=\beta_{2} \cos \theta_{p}, \\
& \tan \theta_{d}=\tan \theta_{r}=q_{1} / k_{0}, \quad \tan \theta_{p}=q_{2} / k_{0},
\end{aligned}
$$

and, as a consequence, two well known formulae (Snellius law of reflection and refraction),

$$
\begin{gathered}
\theta_{d}=\theta_{r}, \\
k_{0}=\beta_{1} \sin \theta_{d}=\beta_{2} \sin \theta_{p},
\end{gathered}
$$




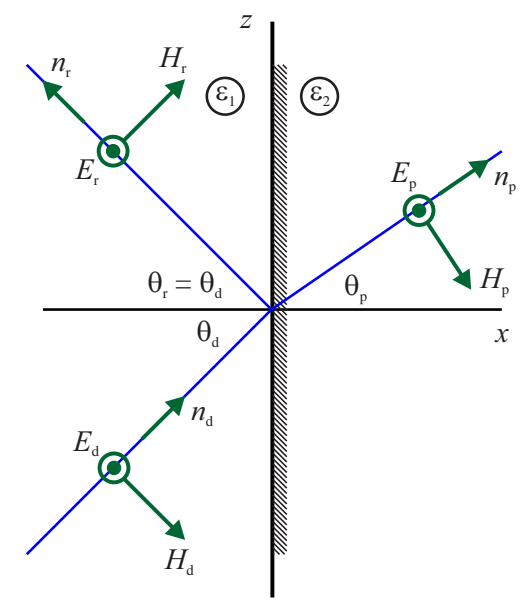

Fig. 1. TE configuration of a plane wave reflection and refraction on the planar dielectric boundary

are obtained.

The reflection and transmission factors in (14) and (24) can be expressed as

$$
\begin{aligned}
& \rho=\frac{q_{1}-q_{2}}{q_{1}+q_{2}}=\frac{\cos \theta_{d}-\sqrt{\varepsilon_{2} / \varepsilon_{1}-\sin ^{2} \theta_{d}}}{\cos \theta_{d}+\sqrt{\varepsilon_{2} / \varepsilon_{1}-\sin ^{2} \theta_{d}}}, \\
& \tau=\frac{2 q_{1}}{q_{1}+q_{2}}=\frac{2 \cos \theta_{d}}{\cos \theta_{d}+\sqrt{\varepsilon_{2} \varepsilon_{1}-\sin ^{2} \theta_{d}}} .
\end{aligned}
$$

The pertaining magnetic intensity vectors of homogeneous plane waves are given by

$$
\begin{aligned}
& \widehat{\mathbf{H}}_{d}(\boldsymbol{r})=\left(\boldsymbol{n}_{d} \times \boldsymbol{u}_{y}\right)\left(E_{0} / Z_{1}\right) \exp \left(-j \beta_{1} \boldsymbol{n}_{d} \cdot \boldsymbol{r}\right), \\
& \widehat{\mathbf{H}}_{r}(\boldsymbol{r})=\left(\boldsymbol{n}_{r} \times \boldsymbol{u}_{y}\right)\left(\rho E_{0} / Z_{1}\right) \exp \left(-j \beta_{1} \boldsymbol{n}_{r} \cdot \boldsymbol{r}\right), \\
& \widehat{\mathbf{H}}_{p}(\boldsymbol{r})=\left(\boldsymbol{n}_{p} \times \mathbf{u}_{y}\right)\left(\tau E_{0} / Z_{2}\right) \exp \left(-j \beta_{2} \mathbf{n}_{p} \cdot \boldsymbol{r}\right),
\end{aligned}
$$

where $Z_{1}=\sqrt{\mu / \varepsilon_{1}}$ and $Z_{2}=\sqrt{\mu / \varepsilon_{2}}$ is the wave impedance of the respective media.

The overall electromagnetic field in medium $\varepsilon_{1}$ is given by the electric intensity vector

$$
\widehat{\mathbf{E}}_{1}(\boldsymbol{r})=\widehat{\mathbf{E}}_{d}(\boldsymbol{r})+\widehat{\mathbf{E}}_{r}(\boldsymbol{r})=\widehat{\mathbf{E}}_{1}(x) \exp \left(-j k_{0} z\right),
$$

where $\widehat{\mathbf{E}}_{1}(x)$ is given by (18), and by the magnetic intensity vector

$$
\widehat{\mathbf{H}}_{1}(\boldsymbol{r})=\widehat{\mathbf{H}}_{d}(\boldsymbol{r})+\widehat{\mathbf{H}}_{r}(\boldsymbol{r})=\widehat{\mathbf{H}}_{1}(x) \exp \left(-j k_{0} z\right),
$$

where $\widehat{\mathbf{H}}_{1}(x)$ is given by (19).

The mean value of the power flow density $\left\langle\mathbf{P}_{1}(x)\right\rangle$ in the medium $\varepsilon_{1}$ is given by (20). The mean value of the power flow density $\left\langle\mathbf{P}_{2}(x)\right\rangle$ in medium $\varepsilon_{2}$ is given by (23) for the electric and magnetic intensity vectors

$$
\begin{aligned}
\widehat{\mathbf{E}}_{2}(\boldsymbol{r}) & =\widehat{\mathbf{E}}_{p}(\boldsymbol{r})=\widehat{\mathbf{E}}_{2}(x) \exp \left(-j k_{0} z\right), \\
\widehat{\mathbf{H}}_{2}(\boldsymbol{r}) & =\widehat{\mathbf{H}}_{p}(\boldsymbol{r})=\widehat{\mathbf{H}}_{2}(x) \exp \left(-j k_{0} z\right),
\end{aligned}
$$

where $\widehat{\mathbf{E}}_{2}(x)$ and $\widehat{\mathbf{H}}_{2}(x)$ are given by $(21)$ and (22).

\section{Critical angle incidence}

Let us consider the case $\varepsilon_{1}>\varepsilon_{2}$, This represents in optics the so-called internal reflection since the incident and reflected wave are $e g$ in glass material $\left(\varepsilon_{1}>\varepsilon_{0}\right)$ and transmitted wave propagates $e g$ in the air $\left(\varepsilon_{2} \approx \varepsilon_{0}\right)$. For the so-called critical angle of incidence $\sin \theta_{d}=\sin \theta_{c}$, where

$$
\sin \theta_{c}=\sqrt{\varepsilon_{2} / \varepsilon_{1}}
$$

one obtains from (41) $\sin \theta_{p}=1$ and from (42), (43) $\rho=1, \tau=2$ as well as from (39) $\mathbf{n}_{p}=\boldsymbol{u}_{z}$, further $q_{1}=\sqrt{\beta_{1}^{2}-\beta_{2}^{2}}, k_{0}=\beta_{2}$, and finally $q_{2}=0$. This is exactly the case described by $(32)-(35)$.

Thus, in the case $\sin \theta_{d}=\sin \theta_{c}=\sqrt{\varepsilon_{2} / \varepsilon_{1}}$, in the halfspace $x<0$, as a consequence of the interference of the incident and reflected wave, a non-homogeneous plane wave propagates in $z$ direction, while in the halfspace $x>0$ propagates a simple homogeneous plane wave, both with the phase velocity $v_{f}=\omega_{0} / \beta_{2}$. The power flow through the boundary $x=0$ is zero.

\section{Total internal reflection}

If angle of incidence $\theta_{d}$ is larger then the critical angle $\theta_{c}$, ie $\theta_{d}>\theta_{c}$ then (41) yields

$$
\sin \theta_{p}=\sin \theta_{d} / \sin \theta_{c}>1
$$

This can be mathematically resolved by introducing the complex angle $\theta_{p}=\pi / 2+j \varsigma$.

Then

$$
\sin \theta_{p}=\sin (\pi / 2+j \varsigma)=\cosh \varsigma=\sin \theta_{d} / \sin \theta_{c}>1,
$$

and $\cos \theta_{p}$ becomes purely imaginary

$$
\cos \theta_{p}=\cos (\pi / 2+j \varsigma)=-j \sinh \varsigma
$$

It also means that $\mathbf{n}_{p}$ becomes a complex vector

$$
\mathbf{n}_{p}=-j \sinh \varsigma \boldsymbol{u}_{x}+\cosh \varsigma \boldsymbol{u}_{z},
$$

ie $\beta_{1}>k_{0}>\beta_{2}$, and $\varkappa$ in (27) is expressed as

$$
\varkappa=\sqrt{k_{0}^{2}-\beta_{2}^{2}}=\beta_{1} \sqrt{\sin ^{2} \theta_{d}-\sin ^{2} \theta_{c}} .
$$

For the reflection factor one obtains

$$
\rho=\frac{\cos \theta_{d}+j \sqrt{\sin ^{2} \theta_{d}-\sin ^{2} \theta_{c}}}{\cos \theta_{d}-j \sqrt{\sin ^{2} \theta_{d}-\sin ^{2} \theta_{c}}}=\exp (j 2 \Psi),
$$




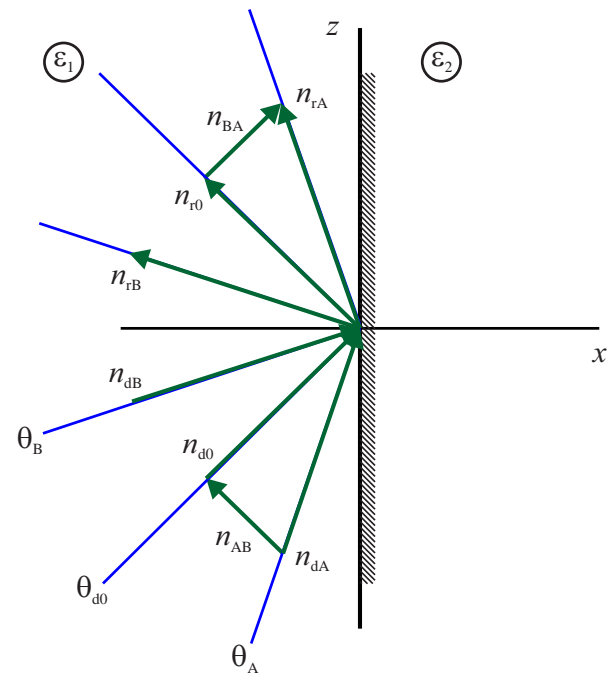

Fig. 2. Definition of the $\mathbf{n}_{d A}, \mathbf{n}_{d B}, \mathbf{n}_{d 0}, \mathbf{n}_{r A}, \mathbf{n}_{r B}, \mathbf{n}_{r 0}$ vectors and pertaining $\theta_{a}, \theta_{b}, \theta_{d 0}$ angles

with alternative expression to that in $(27)$

$$
\cos \Psi=\cos \theta_{d} / \cos \theta_{c}
$$

Thus, the complex reflection coefficient has the magnitude equal to one, $|\rho|=1$, and the phase $2 \Psi$. This is exactly the case described by (27).

For the fields in $\varepsilon_{1}$, and $\varepsilon_{2}$, regions one obtains

$$
\begin{aligned}
\widehat{\mathbf{E}}_{1,2}(\boldsymbol{r}) & =\widehat{\mathbf{E}}_{1,2}(x) \exp \left(-j k_{0} z\right), \\
\widehat{\mathbf{H}}_{1,2}(\boldsymbol{r}) & =\widehat{\mathbf{H}}_{1,2}(x) \exp \left(-j k_{0} z\right),
\end{aligned}
$$

where $\widehat{\mathbf{E}}_{1}(x), \widehat{\mathbf{E}}_{2}(x), \widehat{\mathbf{H}}_{1}(x)$, and $\widehat{\mathbf{H}}_{2}(x)$ are given by $(25)-(29)$.

As already pointed out, mean values of the power flow densities have only components parallel with the boundary (ie the wave power flows exclusively along the boundary with no power passing through the boundary), are in the respective media given by (30) and (31) and are continuous on the boundary plane.

\section{Total internal reflection of an nonhomogeneous plane wave, Goos-Hänchen shift and penetration of the power flow density}

Let us now consider an interference of two incident homogeneous TE-polarised plane waves propagating in directions given by $\mathbf{n}_{d A}$ and $\mathbf{n}_{d B}$

$\widehat{\mathbf{E}}_{d}(\boldsymbol{r})=\mathbf{u}_{y} E_{0} \exp \left(-j \beta_{1} \mathbf{n}_{d A} \cdot \boldsymbol{r}\right)+\mathbf{u}_{y} E_{0} \exp \left(-j \beta_{1} \mathbf{n}_{d B} \cdot \boldsymbol{r}\right)$,

where $\boldsymbol{n}_{d A}=\cos \theta_{A} \boldsymbol{u}_{x}+\sin \theta_{A} \boldsymbol{u}_{z}, \boldsymbol{n}_{d B}=\cos \theta_{B} \boldsymbol{u}_{x}+$ $\sin \theta_{B} \boldsymbol{u}_{z}$, assuming $\theta_{A}>\theta_{B}$.

Introducing two perpendicular non-unit vectors $\boldsymbol{n}_{d 0}$ and $\boldsymbol{n}_{A B}$, Fig. 2, by

$\mathbf{n}_{d 0}=\left(\mathbf{n}_{d A}+\mathbf{n}_{d B}\right) / 2=\cos \theta_{A B}\left(\cos \theta_{d 0} \boldsymbol{u}_{x}+\sin \theta_{d 0} \boldsymbol{u}_{z}\right)$, $\mathbf{n}_{A B}=\left(\boldsymbol{n}_{d A}-\mathbf{n}_{d B}\right) / 2=\sin \theta_{A B}\left(-\sin \theta_{d 0} \boldsymbol{u}_{x}+\cos \theta_{d 0} \boldsymbol{u}_{z}\right)$, where $\theta_{d 0}=\left(\theta_{A}+\theta_{B}\right) / 2, \theta_{A B}=\left(\theta_{A}-\theta_{B}\right) / 2$, one may rewrite (58) as

$$
\widehat{\mathbf{E}}_{d}(\boldsymbol{r})=\boldsymbol{u}_{y} 2 E_{0} \cos \left(\beta_{1} \boldsymbol{n}_{A B} \cdot \boldsymbol{r}\right) \exp \left(-j \beta_{1} \boldsymbol{n}_{d 0} \cdot \boldsymbol{r}\right)
$$

$i e$ as an inhomogeneous plane having in direction $\mathbf{n}_{A B}$, perpendicular to the propagation direction $\boldsymbol{n}_{d 0}$, a nonconstant, cos-like, amplitude pattern of the wavefront

$$
\begin{aligned}
& 2 E_{0} \cos \left(\beta_{1} \mathbf{n}_{A B} \cdot \boldsymbol{r}\right)= \\
& 2 E_{0} \cos \left\{\beta_{1} \sin \theta_{A B}\left(-x \sin \theta_{d 0}+z \cos \theta_{d 0}\right)\right\},
\end{aligned}
$$

with the period $\lambda_{q}=2 \pi / \beta_{1} \sin \theta_{A B}$. This plane wave propagates in direction $\boldsymbol{n}_{d 0}$ with the propagation factor $\exp \left(-j \beta_{1} \boldsymbol{n}_{d 0} \cdot \boldsymbol{r}\right)$ equal to

$$
\exp \left\{-j \beta_{1} \cos \theta_{A B}\left(x \cos \theta_{d 0}+z \sin \theta_{d 0}\right)\right\},
$$

thus with the wavenumber $\beta_{1} \cos \theta_{A B}$, ie with the phase velocity $v_{f}=\omega_{0} / \beta_{1} \cos \theta_{A B}$ higher than the velocity of light in the given media $c_{1}=\omega_{0} / \beta_{1}$.

If both directions $\boldsymbol{n}_{d A}$ and $\boldsymbol{n}_{d B}$ fulfil the condition of the total internal reflection $\theta_{A}, \theta_{B}>\theta_{c}$, then for the resulting reflected wave one obtains

$$
\begin{array}{r}
\widehat{\mathbf{E}}_{r}(\boldsymbol{r})=\boldsymbol{u}_{y} E_{0}\left\{\exp \left(2 j \Psi_{A}\right) \exp \left(-j \beta_{1} \boldsymbol{n}_{r A} \cdot \boldsymbol{r}\right)+\right. \\
\left.\exp \left(2 j \Psi_{B}\right) \exp \left(-j \beta_{1} \mathbf{n}_{r B} \cdot \boldsymbol{r}\right)\right\},
\end{array}
$$

where the reflection factors $\exp \left(2 j \Psi_{A}\right)$ and $\exp \left(2 j \Psi_{B}\right)$ pertain to the angles of incidence $\theta_{A}$ and $\theta_{B}$ respectively, and

$$
\begin{aligned}
& \mathbf{n}_{r A}=-\cos \theta_{A} \boldsymbol{u}_{x}+\sin \theta_{A} \boldsymbol{u}_{z}, \\
& \mathbf{n}_{r B}=-\cos \theta_{B} \boldsymbol{u}_{x}+\sin \theta_{B} \boldsymbol{u}_{z} .
\end{aligned}
$$

Denoting similarly as above

$\mathbf{n}_{r 0}=\left(\mathbf{n}_{r A}+\mathbf{n}_{r B}\right) / 2=\cos \theta_{A B}\left(-\cos \theta_{d 0} \boldsymbol{u}_{x}+\sin \theta_{d 0} \boldsymbol{u}_{z}\right)$, $\boldsymbol{n}_{B A}=\left(\boldsymbol{n}_{r A}-\mathbf{n}_{r B}\right) / 2=\sin \theta_{A B}\left(\sin \theta_{d 0} \boldsymbol{u}_{x}+\cos \theta_{d 0} \boldsymbol{u}_{z}\right)$,

formula (61) can be rewritten into the form

$$
\begin{aligned}
\widehat{\mathbf{E}}_{r}(\boldsymbol{r})=\boldsymbol{u}_{y} 2 E_{0} \cos \left(\beta_{1} \boldsymbol{n}_{B A} \cdot \boldsymbol{r}-\Psi_{A B}\right) \times \\
\exp \left\{-j\left(\beta_{1} \boldsymbol{n}_{r 0} \cdot \boldsymbol{r}-\Psi_{0}\right)\right\},
\end{aligned}
$$

where $\Psi_{0}=\Psi_{A}+\Psi_{B}$ and $\Psi_{A B}=\Psi_{A}-\Psi_{B}$.

The cos-like amplitude pattern of the reflected wave is now given by

$$
\begin{aligned}
& 2 E_{0} \cos \left(\beta_{1} \mathbf{n}_{B A} \cdot \boldsymbol{r}-\Psi_{A B}\right)= \\
& 2 E_{0} \cos \left(\beta_{1} \sin \theta_{A B}\left[\sin \theta_{d 0} x+\cos \theta_{d 0} z\right]-\Psi_{A B}\right),
\end{aligned}
$$

with the same period as the amplitude pattern of the incident wave but shifted in the direction of $\boldsymbol{n}_{B A}$ by

$$
s=\Psi_{A B} / \beta_{1} \sin \theta_{A B} .
$$




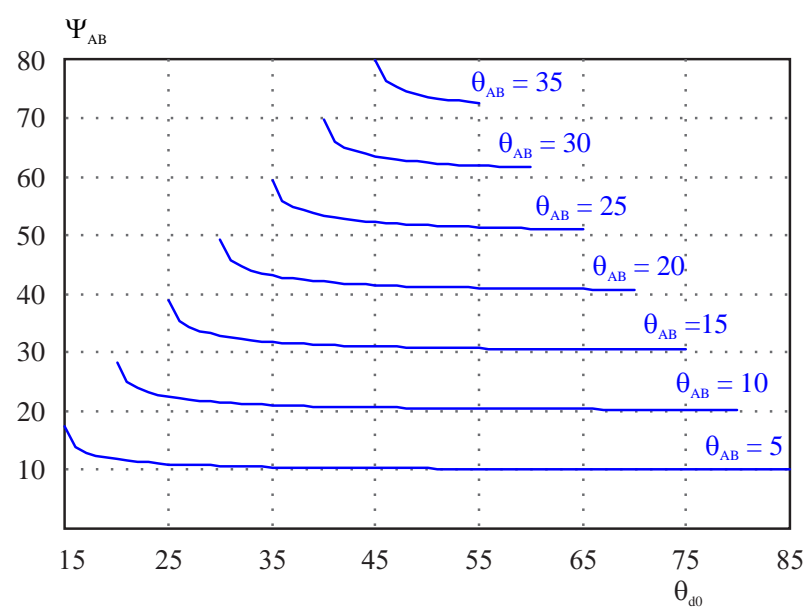

Fig. 3. Dependence of $\Psi_{A B}$ on $\theta_{d 0}$ for $\theta_{c}=10^{\circ}$ and various $\theta_{A B}$ (in degrees)

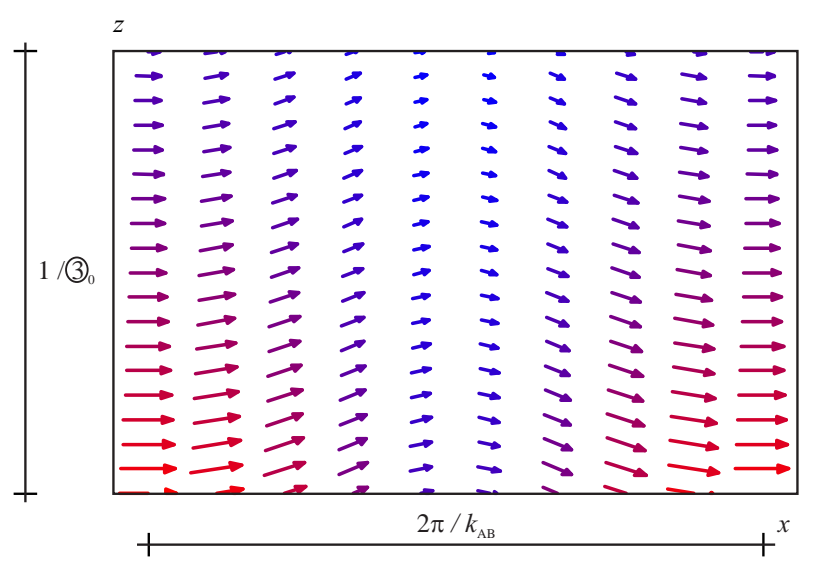

Fig. 5. Schematic illustration of the power flow density $\left\langle\mathbf{P}_{2}\right\rangle$ penetration into medium with $\varepsilon_{2}$

This effect is called the Goos-Hänchen shift. Along the boundary plane $x=0$ this shift equals

$$
s_{z}=s / \cos \theta_{d 0}=\Psi_{A B} / \beta_{1} \sin \theta_{A B} \cos \theta_{d 0} .
$$

The link between $\Psi_{0}=\Psi_{A}+\Psi_{B}, \Psi_{A B}=\Psi_{A}-\Psi_{B}$ and $2 \theta_{d 0}=\theta_{A}+\theta_{B}, 2 \theta_{A B}=\theta_{A}-\theta_{B}$ can be easily established in the form

$$
\begin{aligned}
& \cos \Psi_{0}=\left\{\cos \left(2 \theta_{d 0}\right)+\cos \left(2 \theta_{A B}\right)-\Delta\right\} / 2 \cos ^{2} \theta_{c}, \\
& \cos \Psi_{A B}=\left\{\cos \left(2 \theta_{d 0}\right)+\cos \left(2 \theta_{A B}\right)+\Delta\right\} / 2 \cos ^{2} \theta_{c}
\end{aligned}
$$

where

$$
\begin{aligned}
\Delta=\left[\cos ^{2}\left(2 \theta_{d 0}\right)\right. & +\cos ^{2}\left(2 \theta_{A B}\right)-\sin ^{2}\left(2 \theta_{c}\right)- \\
& \left.2 \cos \left(2 \theta_{d 0}\right) \cos \left(2 \theta_{A B}\right) \cos \left(2 \theta_{c}\right)\right]^{1 / 2} .
\end{aligned}
$$

The dependence of $\Psi_{A B}$ on $\theta_{d 0}$ and $\theta_{A B}$ is depicted in Figs. 3 and 4 , for the interval $\theta_{d 0} \in\left(\theta_{c}+\theta_{A B}, \pi / 2-\theta_{A B}\right)$, with $\theta_{A B}$ as a parameter of the series of curves. It is seen in Fig. 3 that for $\theta_{c}=10^{\circ}$ except of $\theta_{B}$ being very close to $\theta_{c}$ the phase angle $\Psi_{A B}$ converges to the angle very

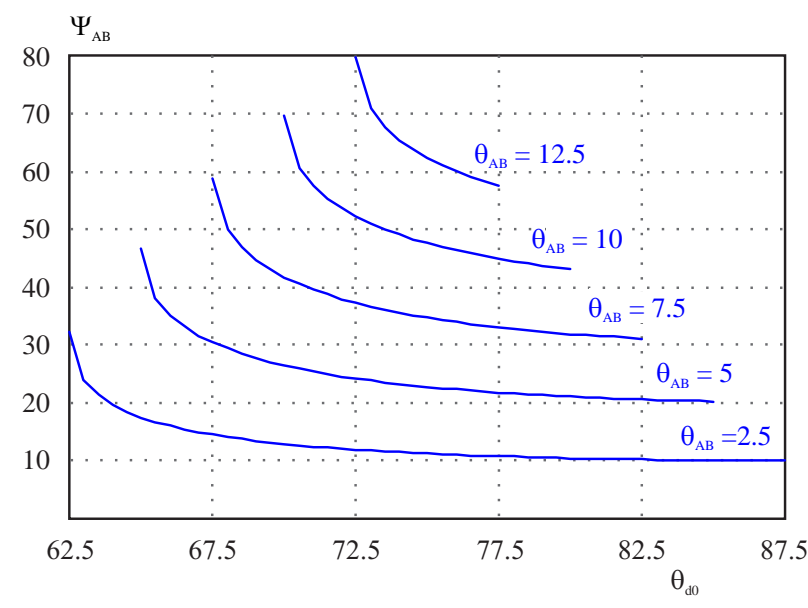

Fig. 4. Dependence of $\Psi_{A B}$ on $\theta_{d 0}$ for $\theta_{c}=60^{\circ}$ and various $\theta_{A B}$ (in degrees)

close to $2 \theta_{A B}$. For $\theta=60^{\circ}$ in Fig. 4 the range of the phase angles $\Psi_{A B}$ as a function of $\theta_{d 0}$ is much broader, for $\theta_{d 0} \rightarrow \pi / 2-\theta_{A B}$ having the tendency to converge to the angle $4 \theta_{A B}$.

For $\theta_{A B}=\pi / 4-\theta_{c} / 2$ there is only one value of $\theta_{d 0}=\pi / 4+\theta_{c} / 2$ and $\Psi_{A B}=\pi / 2$ representing the maximum value of $\Psi_{A B}$ attainable.

The overall $E$-field in the second media with permittivity $\varepsilon_{2}$ equals

$$
\begin{aligned}
\widehat{\mathbf{E}}_{2}(\boldsymbol{r})= & \mathbf{u}_{y} 2 E_{0}\left\{\cos \Psi_{A} \exp \left(-\varkappa_{A} x\right) \exp \left(-j\left[k_{A} z-\Psi_{A}\right]\right)\right. \\
& \left.+\cos \Psi_{B} \exp \left(-\varkappa_{B} x\right) \exp \left(-j\left[k_{A} z-\Psi_{B}\right]\right)\right\}
\end{aligned}
$$

where $k_{A, B}=\beta_{1} \cos \theta_{d A, d B}, \varkappa_{A, B}=\sqrt{k_{A, B}^{2}-\beta_{2}^{2}}$. For the $H$-field in the second media one obtains

$$
\begin{aligned}
\widehat{\mathbf{H}}_{2}(\boldsymbol{r})=-\left(2 E_{0} / \omega_{0} \mu\right)\left\{\cos \Psi_{A}\left(j \varkappa_{A} \boldsymbol{u}_{z}+k_{A} \boldsymbol{u}_{x}\right)\right) \times \\
\exp \left(-\varkappa_{A} x\right) \exp \left(-j\left[k_{A} z-\Psi_{A}\right]\right)+\cos \Psi_{B}\left(j \varkappa_{B} \boldsymbol{u}_{z}+k_{B} \mathbf{u}_{x}\right) \\
\times \exp \left(-\varkappa_{B} x\right) \exp \left(-j\left[k_{B} z-\Psi_{B}\right]\right)
\end{aligned}
$$

The $z$-component of the mean value of the power flow density $\left\langle\mathbf{P}_{2}\right\rangle$ in the medium $\varepsilon_{2}$ is

$$
\begin{gathered}
\left\langle P_{2 z}\right\rangle=\left(2 E_{0}^{2} / \omega_{0} \mu\right)\left\{k_{A} \cos ^{2} \Psi_{A} \exp \left(-2 \varkappa_{A} x\right)+\right. \\
k_{B} \cos ^{2} \Psi_{B} \exp \left(-2 \varkappa_{B} x\right)+
\end{gathered}
$$$$
\left.k_{0} \cos \Psi_{A} \cos \Psi_{B} \exp \left(-\varkappa_{0} x\right) \cos \left(k_{A B} z-\Psi_{A B}\right)\right\} \text {, }
$$

where $\varkappa_{0}=\varkappa_{A}+\varkappa_{B}, k_{0}=k_{A}+k_{B}, k_{A B}=k_{A}-k_{B}=$ $\beta_{1} \cos \theta_{d 0} \sin \theta_{A B}$.

The $x$-component of $\left\langle\mathbf{P}_{2}\right\rangle$ equals

$$
\begin{aligned}
\left\langle P_{2 x}\right\rangle=-\left(2 E_{0}^{2} / \omega_{0} \mu\right) k_{A B} \cos \Psi_{A} \cos \Psi_{B} & \\
& \times \exp \left(-\varkappa_{0} x\right) \sin \left(k_{A B} z-\Psi_{A B}\right) .
\end{aligned}
$$

The $z$-component of the power flow on the boundary is given by

$$
\begin{aligned}
\left.\left\langle P_{2 z}\right\rangle\right|_{x=0}= & \left(2 E_{0}^{2} / \omega_{0} \mu\right)\left\{k_{A} \cos ^{2} \Psi_{A}+k_{B} \cos ^{2} \Psi_{B}+\right. \\
& \left.k_{0} \cos \Psi_{A} \cos \Psi_{B} \cos \left(k_{A B} z-\Psi_{A B}\right)\right\}
\end{aligned}
$$


and oscillates between the minimum and maximum value

$\left(E_{0}^{2} / \mu\right)\left\{k_{A B}\left[\cos ^{2} \Psi_{A}-\cos ^{2} \Psi_{B}\right]+k_{0}\left[\cos \Psi_{A} \pm \cos \Psi_{B}\right]^{2}\right\}$.

The power flow through the boundary is given by

$$
\begin{aligned}
& \left.\left\langle P_{2 x}\right\rangle\right|_{x=0}= \\
& \quad-\left(2 E_{0}^{2} / \mu\right) k_{A B} \cos \Psi_{A} \cos \Psi_{B} \sin \left(k_{A B} z-\Psi_{A B}\right)
\end{aligned}
$$

and oscillates between the maximum positive and negative values $\pm\left(2 E_{0}^{2} / \mu\right) k_{A B} \cos \Psi_{A} \cos \Psi_{B}$ as given by the term $\sin \left(k_{A B} z-\Psi_{A B}\right.$ in (74). Observe that in the points, where transversal component $\left.\left\langle P_{2 x}\right\rangle\right|_{x=0}$ is zero, the longitudinal component $\left.\left\langle P_{2 z}\right\rangle\right|_{x=0}$ reaches its maximum, or minimum.

It means that there are portions of the boundary plane where the power penetrates from the medium with $\varepsilon_{1}$ into the medium with $\varepsilon_{2}$ and in other portions of the boundary it returns back from the medium with $\varepsilon_{2}$ into the medium with $\varepsilon_{1}$. This is the fundamental difference between the homogeneous and non-homogeneous plane wave case and it is the real physical cause of the GoosHänchen shift. In case of the homogeneous plane wave, the power flows along the boundary plane only, the reflected wave obtains the phase shift $\Psi$ but no power penetration into the second medium occurs. In case of the non-homogeneous plane wave this phase shift is transformed into the spatial shift with the power being transferred through the second medium. Observe that the Goos-Hänchen shift on the $z$-axis (65) equals exactly the shift of the sin function in (74)

$$
s_{z}=s / \cos \theta_{d}=\Psi_{A B} / \beta_{1} \sin \theta_{A B} \cos \theta_{d}=\Psi_{A B} / k_{A B} .
$$

\section{Total internal reflection of a monochromatic beam wave}

Let us instead of incident plane waves consider a twodimensional TE-polarised beam wave

$$
\widehat{\mathbf{E}}_{d}(\boldsymbol{r})=\widehat{\mathbf{E}}_{d}(\xi, \zeta)=\mathbf{u}_{y} \widehat{E}_{y}(\xi, \zeta)=\mathbf{u}_{y} E_{0} \varphi_{d}(\xi, \zeta)
$$

propagating along the longitudinal $\zeta$-axis identical with the direction of the $\boldsymbol{n}_{d 0}$ under the angle-of-incidence $\theta_{d 0}$ to the $x$-axis, with the amplitude profile of the beam wave along the transversal $\xi$-axis identical with the direction of the $\mathbf{n}_{A B}$, perpendicular to $\zeta$-axis, where

$$
\begin{aligned}
& \xi=-\left(x+x_{0}\right) \sin \theta_{d 0}+\left(z-z_{0}\right) \cos \theta_{d 0} \\
& \zeta=\left(x+x_{0}\right) \cos \theta_{d 0}+\left(z-z_{0}\right) \sin \theta_{d 0}
\end{aligned}
$$

The origin of the $(\xi, \zeta)$ coordinate system is thus placed in the point $\left(-x_{0}, z_{0}\right)$ of the $(x, z)$ coordinate system.

The beam-wave-profile function $\varphi_{d}(\xi, \zeta)$ is given in the plane $\zeta=0$ by the spatial initial condition $\varphi_{d 0}(\xi)$, ie $\left.\varphi_{d}(\xi, \zeta)\right|_{\zeta=0}=\varphi_{d 0}(\xi)$.
In fact the wave given in (59) is an example of such a wave, however with cos-like amplitude pattern, ie

$$
\widehat{\mathbf{E}}_{d}(\boldsymbol{r})=\boldsymbol{u}_{y} 2 E_{0} \cos \left(q_{0} \xi\right) \exp \left(-j k_{0} \zeta\right)
$$

where $\varphi_{d}(\xi, \zeta)=2 \cos \left(q_{0} \xi\right) \exp \left(-j k_{0} \zeta\right), q_{0}=\beta_{1} \sin \theta_{A B}$, $k_{0}=\sqrt{\beta_{1}^{2}-q_{0}^{2}}=\beta_{1} \cos \theta_{A B}$.

As shown in [7], the profile pattern $\varphi_{d}(\xi, \zeta)$ can be generally expressed in form of an integral summation of infinitesimal plane waves $\Phi_{d}(q, \zeta) \exp (-j q \xi) d q$ with the spectral density

$$
\Phi_{d}(q, \zeta)=\Phi_{d 0}(q) \exp \left\{-j \zeta \sqrt{\beta_{1}^{2}-q^{2}}\right\}
$$

where

$$
\Phi_{d 0}(q)=\frac{1}{2 \pi} \int_{-\infty}^{\infty} \varphi_{d 0}(\xi) \exp (j q \xi) \mathrm{d} \xi
$$

is the Fourier transform of the initial profile $\varphi_{d 0}(\xi)$, ie

$$
\varphi_{d}(\xi, \zeta)=\int_{-\infty}^{\infty} \Phi_{d 0}(q) \exp \left\{-j\left(q \xi+\zeta \sqrt{\beta_{1}^{2}-q^{2}}\right)\right\} \mathrm{d} q
$$

The infinitesimal plane wave components belonging to $\Phi_{d}(q, \zeta)$ propagate spanning the angles $\theta_{q}=\arcsin \left(q / \beta_{1}\right)$ with respect to the beam axis.

The inhomogeneous plane wave in (76) is just an example of (79) with a singular spatial-frequency component, ie $\Phi_{d 0}(q)=\delta\left(q-q_{0}\right)+\delta\left(q+q_{0}\right)$, where $\delta(q)$ is the Dirac delta-function.

If considering spatially-bandlimited beams, ie if $\Phi_{d 0}(q)=0$ for $q>q_{\max }$, then, if $q_{\max } \ll \beta_{1}$, the Fresnel (or parabolic) approximation of $\sqrt{\beta_{1}^{2}-q^{2}}$

$$
\sqrt{\beta_{1}^{2}-q^{2}} \approx \beta_{1}-q^{2} / 2 \beta_{1}
$$

can be used in the phase terms of (77) and (79). Then the phase term can be approximately written as

$$
\zeta \sqrt{\beta_{1}^{2}-q^{2}} \approx \beta_{1} \zeta-q^{2} \zeta / 2 \beta_{1}=2 \pi\left\{(\zeta / \lambda)-\left(\zeta / 2 \zeta_{F}\right)\right\}
$$

where the characteristic length $\zeta_{F}$ is defined by $\zeta_{F}=$ $2 \pi \beta_{1} / q^{2}=\lambda_{q}^{2} / \lambda$ and $\lambda=2 \pi / \beta_{1}, \lambda_{q}=2 \pi / q$. Until the propagation path $\zeta$ is much smaller than $\zeta_{F \text { min }}$, ie $\zeta / \zeta_{F \text { min }} \ll 1$, where $\zeta_{F \text { min }}$ and $\lambda_{q \text { min }}$ correspond to $q_{\max }$, the beam wave behaviour mimics the propagation of a plane wave having nearly planar wavefront with small changes of the beam profile. For $\zeta / \zeta_{F \text { min }} \gg 1$ the beam wave diverges, the beam-profile spreads-out, reaching in the limiting case $z \rightarrow \infty$ the form of a spherical wave.

If, in parabolic approximation, the ratio $\zeta_{\max } / \zeta_{F \min }$ (the so called Fresnel number) is invariant for various initial beam widths and various propagation path lengths, then the propagation-characteristics of all various beams are identical.

The beam wave expressed by (79) consists for $|q|>$ $\beta_{1}$ also of evanescent waves. If the beam wave should 


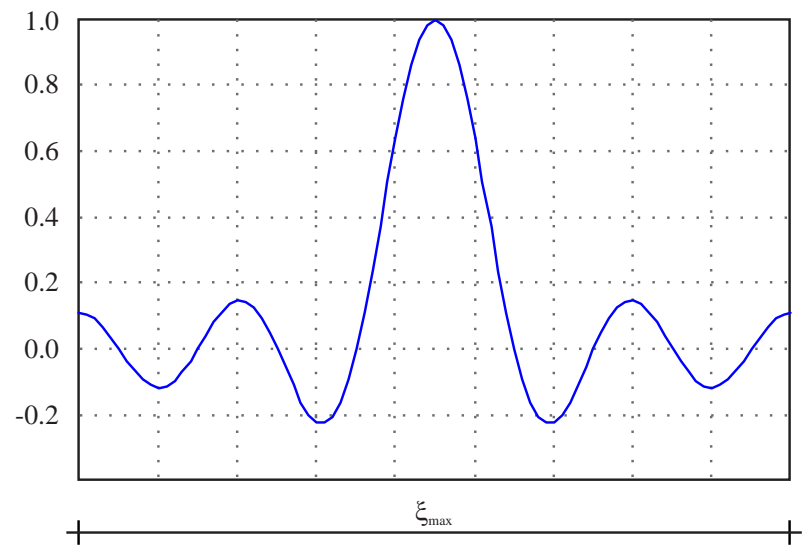

Fig. 6. Initial beam-amplitude profile $\varphi_{d 0}(\xi)=h(\xi)$ for $N=4$

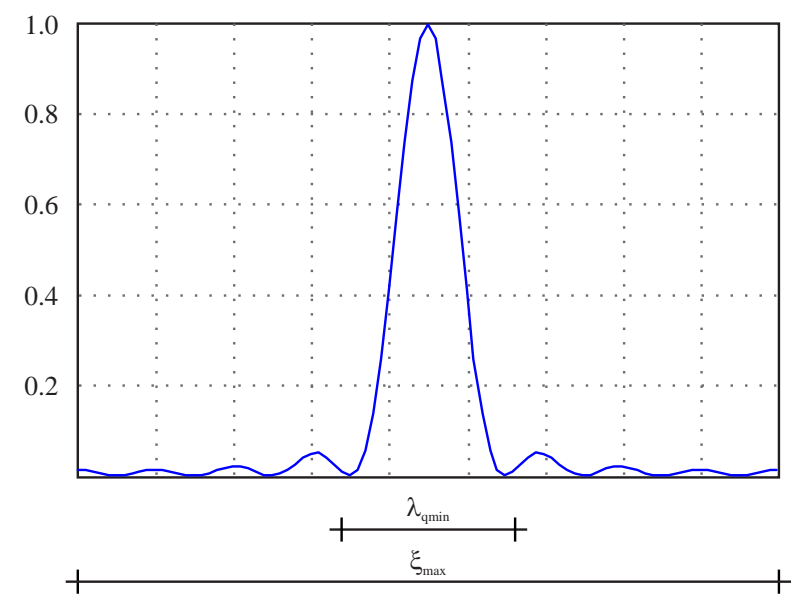

Fig. 7. Initial beam-intensity profile $\left|\varphi_{d 0}(\xi)\right|^{2}$ of Fig. 6

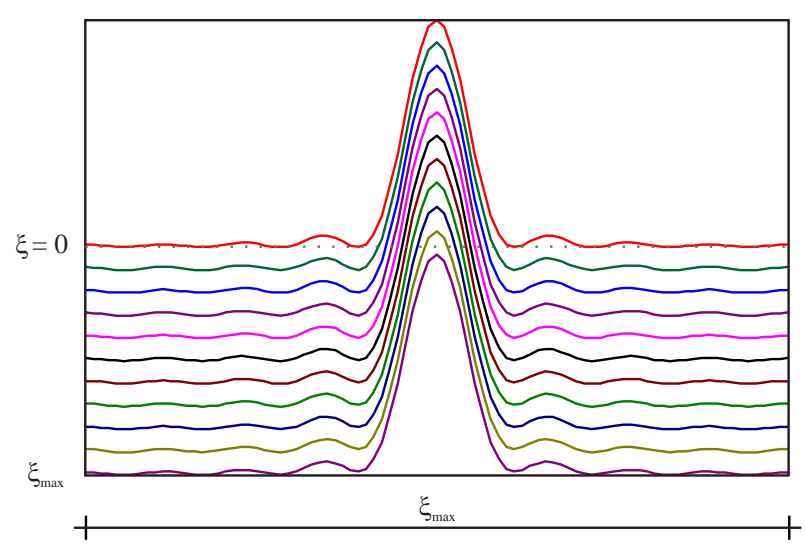

Fig. 8. Incident beam intensity along the propagation path $\zeta \in$ $\left(0, \zeta_{\max }\right)$ for $\zeta_{\max } / \lambda=4, \lambda / \lambda_{q \min }=0.2$, and $\zeta_{\max } / \zeta_{F \max }=0.16$

consist only of homogeneous plane waves then the integration in (79) has to by bounded to interval $q \in$ $\left(-\beta_{1}, \beta_{1}\right)$. To each value of $q$ corresponds the angle of incidence of the pertaining plane wave $\theta=\theta_{d 0}+\theta_{q}$, where $\theta_{q}=\arcsin \left(q / \beta_{1}\right)$ and $\theta_{d 0}$ is the angle of the beam axis spanned with the $x$-axis.

Since we require that all of the spatial spectral components fulfil the condition of the total reflection, then any angle of incidence $\theta$ must be larger than the critical angle, $\theta>\theta_{c}$, and smaller than $\pi / 2$ (grazing incidence). After the substitution $\beta_{1} \sin \left(\theta-\theta_{d 0}\right)=q$ one obtains (79) expressed in terms of incidence angles $\theta$

$$
\begin{aligned}
\varphi_{d}(\xi, \zeta) & =\beta_{1} \int_{\theta_{c}}^{\pi / 2} \Phi_{d 0}(\theta) \exp \left\{-j \zeta \beta_{1} \cos \left(\theta-\theta_{d 0}\right)\right\} \\
& \times \exp \left\{-j \beta_{1} \sin \left(\theta-\theta_{d 0}\right) \xi\right\} \cos \left(\theta-\theta_{d 0}\right) \mathrm{d} \theta
\end{aligned}
$$

In order to have $\theta$ within the angle interval $\theta \in$ $\left(\theta_{c}, \pi / 2\right)$ of the total reflection for the symmetrical position of the beam axis $\theta_{d 0}=\theta_{c} / 2+\pi / 4$, the pertaining $\theta_{q}=\arcsin \left(q / \beta_{1}\right)$ must be from the angle interval $\theta_{q} \in\left(-\theta_{\max }, \theta_{\max }\right), \theta_{\max }=\pi / 4-\theta_{c} / 2$. The bounds of the integral in $(79)$ are then given by $q \in\left(-q_{\max }, q_{\max }\right)$, where

$$
q_{\max }=\beta_{1} \sin \left(\pi / 4-\theta_{c} / 2\right) .
$$

The reflected wave

$$
\widehat{\mathbf{E}}_{r}(\chi, \eta)=\mathbf{u}_{y} \widehat{E}_{y}(\chi, \eta)=\boldsymbol{u}_{y} E_{0} \varphi_{r}(\chi, \eta)
$$

can be obtained from the integral

$$
\begin{aligned}
& \varphi_{r}(\chi, \eta)=\beta_{1} \int_{\theta_{c}}^{\pi / 2} \Phi_{d 0}(\theta) \exp \left\{-j \eta \beta_{1} \cos \left(\theta-\theta_{d 0}\right)+j \Psi_{0}\right\} \\
& \quad \times \exp \left\{-j \beta_{1} \sin \left(\theta-\theta_{d 0}\right) \chi-\Psi_{q}\right\} \cos \left(\theta-\theta_{d 0}\right) \mathrm{d} \theta
\end{aligned}
$$

where the propagation direction is given along the longitudinal $\eta$-axis identical vith the direction of the $\mathbf{n}_{r 0}$, and the amplitude profile of the beam wave is given along the transversal $\chi$-axis identical vith the direction of the $\mathbf{n}_{B A}$, where

$$
\begin{aligned}
& \chi=\left(x-x_{0}\right) \sin \theta_{d 0}+\left(z-z_{0}\right) \cos \theta_{d 0}, \\
& \eta=-\left(x-x_{0}\right) \cos \theta_{d 0}+\left(z-z_{0}\right) \sin \theta_{d 0} .
\end{aligned}
$$

The origin of the coordinate system $(\chi, \eta)$ is placed in the point $\left(x_{0}, z_{0}\right)$, mirrored to the origin of the $(\xi, \zeta)$ coordinate system with respect to the $x=0$ plane. The both phase angles $\Psi_{0}$ and $\Psi_{q}$ in exp-functions i (84) are due to (66) and (67) functions of $\theta_{d 0}$ and $\theta_{q}$.

The refracted evanescent beam wave is given by the integral

$$
\begin{aligned}
& \widehat{\mathbf{E}}_{2}(\boldsymbol{r})=\boldsymbol{u}_{y} 2 \beta_{1} E_{0} \int_{\theta_{c}}^{\pi / 2}\{\cos \Psi \times \\
&\left.\exp (-\varkappa x) \exp (-j[k z-\pi \Psi]) \cos \left(\theta-\theta_{d 0}\right)\right\} \mathrm{d} \theta,
\end{aligned}
$$

where $k=\beta_{1} \cos \theta, \varkappa=\sqrt{k^{2}-\beta_{2}^{2}}, \theta=\theta_{d 0}+\theta_{q}$.

The pertaining $\widehat{\mathbf{H}}$ vectors can be obtained applying (8) to the integrals (81), (84) and (85).

\section{Numerical implementation}

Various numerical implementation aspects of the beamwave-propagation simulation are thoroughly discussed in [7]. Here we briefly review some of them. 


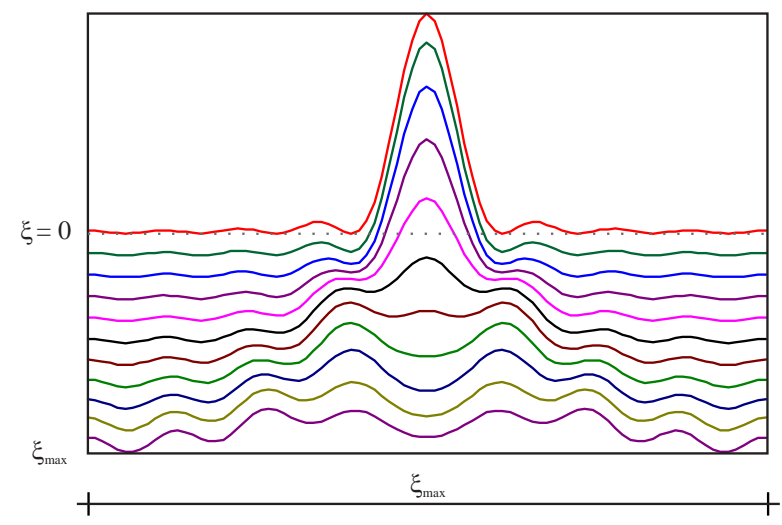

Fig. 9. Incident beam intensity along the propagation path $\zeta \in$ $\left(0, \zeta_{\max }\right)$ for $\zeta_{\max } / \lambda=40, \lambda / \lambda_{q \min }=0.2, \zeta_{\max } / \zeta_{F \min }=16$

In the numerical modelling only the limited number $2 N+1$ (for the simplicity we shall consider the odd number only) of the discrete harmonics of the incident beam wave $\Phi_{d n}(\zeta)=\Phi_{d}\left(q_{n}, \zeta\right)$, instead of continuous $\Phi_{d}(q, \zeta)$ in $(77)$, can be used, where

$\Phi_{d}\left(q_{n}, \zeta\right)=\Phi_{d 0}\left(q_{n}\right) \exp \left(-j \zeta \sqrt{\beta_{1}^{2}-q_{n}^{2}}\right), \quad q_{n}=n \Delta_{q}$, $n \in\{-N, N\}$. Using the Fourier series

$$
\widetilde{\varphi}_{d}(\xi, \zeta)=\sum_{n=-N}^{N} \Phi_{d n}(\zeta) \exp \left(-j n \Delta_{q} \xi\right)
$$

instead of the Fourier transform (79), one obtains a periodic function $\widetilde{\varphi}_{d}(\xi, \zeta)$ approximating $\varphi_{d}(\xi, \zeta)$ on the interval $\left(-\frac{1}{2} \xi_{\max }, \frac{1}{2} \xi_{\max }\right), \xi_{\max }=2 \pi / \Delta_{q}$. In the points $\xi_{k}=k \Delta_{\xi}, k \in\{-N, \ldots, N\}$, where $\Delta_{\xi}=\xi_{\max } /(2 N+1)$, the values $\widetilde{\varphi}_{d k}(\zeta)=\widetilde{\varphi}_{d}\left(\xi_{k}, \zeta\right)$ are obtained as

$$
\widetilde{\varphi}_{d k}(\zeta)=\sum_{n=-N}^{N} \Phi_{d n}(\zeta) \exp \left\{-j \pi k n /\left(N+\frac{1}{2}\right)\right\} .
$$

As known from the theory of Fourier series [8], the values $\widetilde{\varphi}_{d k}(\zeta)=\widetilde{\varphi}_{d}\left(\xi_{k}, \zeta\right)$ are exactly equal to $\varphi_{d}\left(\xi_{k}, \zeta\right)$ only if $\varphi_{d}(\xi, \zeta)$ were equal to zero outside the interval $\left(-\frac{1}{2} \xi_{\max }, \frac{1}{2} \xi_{\max }\right)-$ situation that never occurs due to the diffraction integral (79), even if it were true for $\varphi_{d 0}(\xi)$ in (78). Otherwise the following relation

$$
\widetilde{\varphi}_{d k}(\zeta)=\sum_{\ell=-\infty}^{\infty} \varphi_{d}\left(m \Delta_{\xi}, \zeta\right), m=k+\ell(2 N+1)
$$

between the "exact" $\varphi_{d}\left(\xi_{k}, \zeta\right)$ and "approximate" $\widetilde{\varphi}_{d k}(\zeta)$ values holds. Therefore usually using the finite set of harmonics only the "approximate" discrete values $\widetilde{\varphi}_{d k}(\zeta)$ are obtained.

The formula (87) exactly corresponds to the definition of the inverse discrete Fourier transform. Therefore also

$$
\Phi_{d n}=\frac{1}{2 N+1} \sum_{k=-N}^{N} \widetilde{\varphi}_{d k} \exp \left\{j \pi k n /\left(N+\frac{1}{2}\right)\right\}
$$

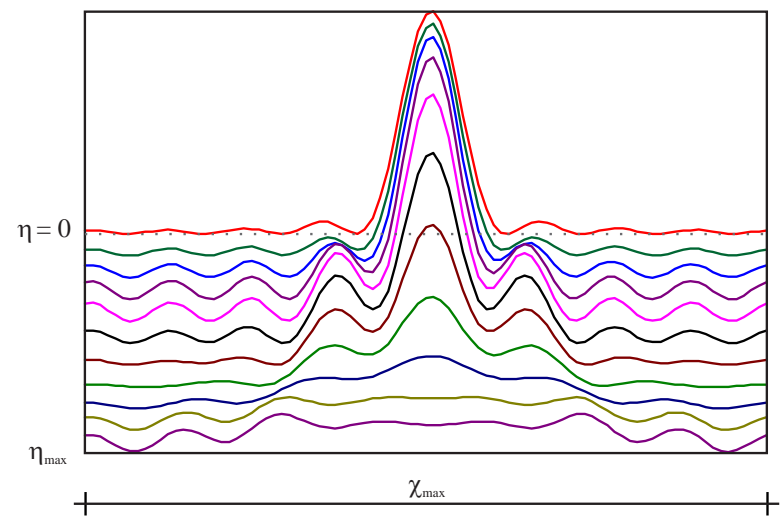

Fig. 10. Reflected beam intensity along the propagation path $\zeta \in$ $\left(0, \zeta_{\max }\right)$ for $\zeta_{\max } / \lambda=40, \lambda / \lambda_{q \min }=0.2, \zeta_{\max } / \zeta_{F \min }=16$

holds. Substituting (89) into (86) after having performed summation with respect to index $n$ yields

$$
\begin{aligned}
& \widetilde{\varphi}_{d}(\xi, \zeta)=\frac{1}{2 N+1} \sum_{k=-N}^{N} \widetilde{\varphi}_{d k}(\zeta) \times \\
& \quad \sin \left\{\left(N+\frac{1}{2}\right) \Delta_{q} \xi-\pi k\right\} / \sin \left\{\frac{1}{2} \Delta_{q} \xi-\frac{\pi k}{2 N+1}\right\} .
\end{aligned}
$$

The last formula (90) in fact means an interpolation of the bandlimited function $\widetilde{\varphi}_{d}(\xi, \zeta)$ between its sampled values $\widetilde{\varphi}_{d k}(\zeta)$ in the sampling points equal to $\xi_{k}=k \Delta_{\xi}$.

The necessity of using the boundary conditions on the boundaries of the computational window $\left(-\frac{1}{2} \xi_{\max }, \frac{1}{2} \xi_{\max }\right)$ leads to either "electric wall periodic boundary condition" where $\Phi_{0(-n)}(\zeta)=-\Phi_{0 n}(\zeta)$ holds, or to "magnetic wall periodic boundary condition", where the $\Phi_{d(-n)}(\zeta)=$ $\Phi_{d n}(\zeta)$ condition is met. As shown in [7] the use of "magnetic wall" condition leads to the lower deterioration of the beam wave spreading due to diffraction effects. Then $\widetilde{\varphi}_{d}(\xi, \zeta)$ instead of (86) can be expressed by

$$
\widetilde{\varphi}_{d}(\xi, \zeta)=\Phi_{d 0}(\zeta)+\sum_{n=1}^{N} 2 \Phi_{d n}(\zeta) \cos \left(n \Delta_{q} \xi\right)
$$

\section{Modeling of the incident and reflected beam wave}

For the numerical investigation of the propagation and total reflection effects we take in place of a initial beam profile $\varphi_{d 0}(\xi)$ directly the kernel function $h(\xi)$ in (90), $\varphi_{d 0}(\xi)=h(\xi)$, where

$$
h(\xi)=\sin \left\{\left(N+\frac{1}{2}\right) \Delta_{q} \xi\right\} /(2 N+1) \sin \left(\frac{1}{2} \Delta_{q} \xi\right) .
$$

Having modeled propagation and reflection of the wave with this profile provides us with the possibility to construct any beam profile by simple superposition in form of (90). The additional advantage is that it possesses 


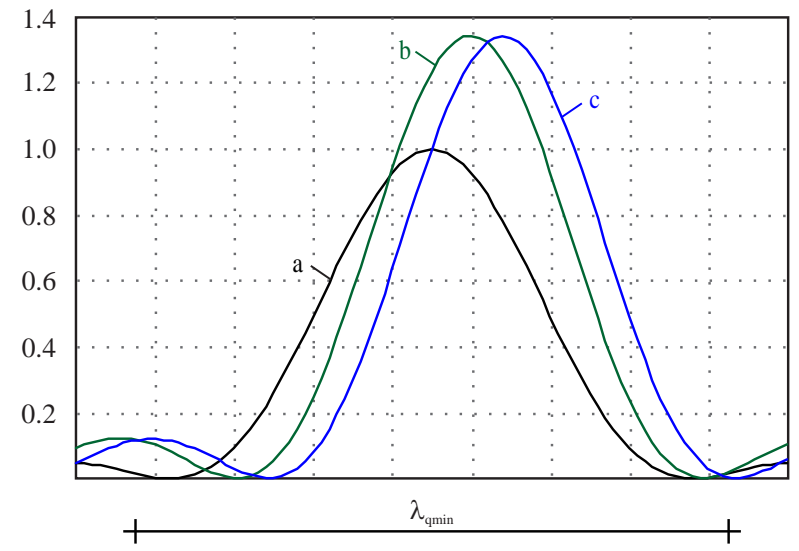

Fig. 11. The central portion (zoomed 10:3) of initial beamintensity profile for $N=4$, of (a) - incident wave (Fig. 8, $\zeta=$ 0 ), (b) - reflected wave (Fig. $10, \eta=0$ ), (c) reflected wave for $\lambda / \lambda_{q \min }=0.36$

the discrete band-limited spatial frequency spectrum with constat amplitude $\mathcal{H}_{n}=1 /(2 N+1), n \in\{-N, N\}$, ie

$$
\begin{array}{r}
h(\xi)=\sum_{n=-N}^{N} \mathcal{H}_{n} \exp \left(-j q_{n} \xi\right)=\frac{1}{2 N+1} \sum_{n=-N}^{N} \exp \left(-j q_{n} \xi\right) \\
=\frac{1}{2 N+1}+\sum_{n=1}^{N} \cos \left(q_{n}(\xi) /\left(N+\frac{1}{2}\right)\right.
\end{array}
$$

where $q_{n}=n \Delta_{q}$.

The propagating incident wave can be easily calculated as

$$
\widetilde{\varphi}_{d}(\xi, \zeta)=\frac{1}{2 N+1} \sum_{n=-N}^{N} \exp \left(-j \zeta \sqrt{\beta_{1}^{2}-q_{n}^{2}}\right) \exp \left(-j q_{n} \xi\right)
$$

or due to the symmetry simply by

$$
\begin{aligned}
\widetilde{\varphi}_{d}(\xi, \zeta) & =\frac{1}{2 N+1} \exp \left(-j \beta_{1} \zeta\right)+ \\
& \sum_{n=1}^{N} \exp \left(-j \zeta \sqrt{\beta_{1}^{2}-q_{n}^{2}}\right) \cos \left(q_{n} \xi\right) /\left(N+\frac{1}{2}\right) .
\end{aligned}
$$

The reflected wave is then given by

$$
\begin{aligned}
& \widetilde{\varphi}_{r}(\chi, \eta)=\frac{1}{2 N+1} \exp \left\{-j\left(\beta_{1} \eta-\Psi_{0}\right)\right\}+ \\
& \frac{2}{2 N+1} \sum_{n=1}^{N} \exp \left\{-j\left(\eta \sqrt{\beta_{1}^{2}-q_{n}^{2}}-\Psi_{n 0}\right)\right\} \cos \left(q_{n} \chi-\Psi_{n}\right)
\end{aligned}
$$

\section{Simulation Results}

As an example we have constructed the initial beam amplitude profile $\widetilde{\varphi}_{d 0}(\xi)=h(\xi)$ accordingly $(92)$ in the plane $\zeta=0$ within the computational window $\xi \in$

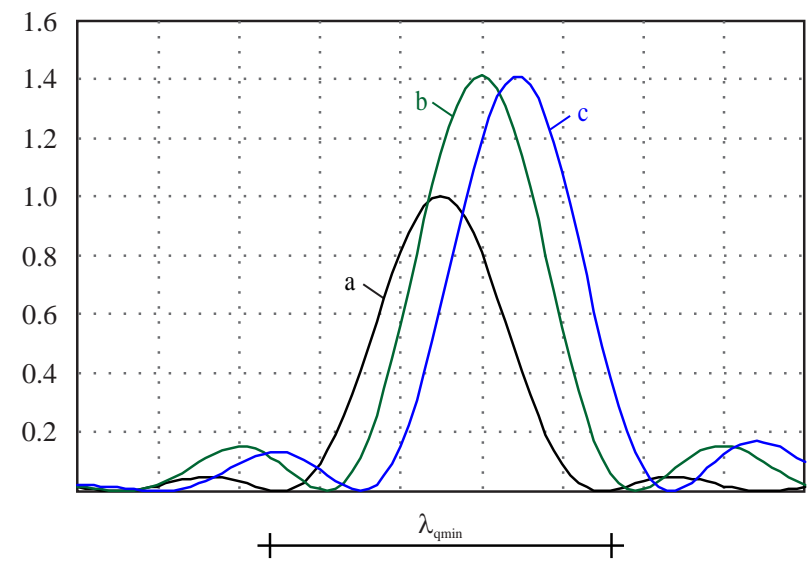

Fig. 12. The central portion (zoomed $10: 3$ ) of the initial beamintensity profile for $N=7$, of (a) incident wave, (b) - reflected wave for $\lambda / \lambda_{q \min }=0.2,(\mathrm{c})-$ reflected wave for $\lambda / \lambda_{q \min }=0.36$

$\left(-\frac{1}{2} \xi_{\max }, \frac{1}{2} \xi_{\max }\right)$ with $N=4$, ie with 9 sampling points as shown in Fig. 6. The sampling points are eight zero points of the profile in Fig. 6 and the central point. The distance of the two central zeros can be considered as a diameter of the beam, ie $2 \xi_{\max } / 9$ in Fig. 6 .

The distribution of intensity is depicted in Fig. 7, showing the good confinement of the beam.

From Fig. 8, where is depicted the propagation along the length $\zeta_{\max } / \lambda=4$, is clearly seen that the wave behaves essentially as a spatially localised plane wave. In the course of further propagation as depicted in Fig. 9 for $\zeta_{\max } / \lambda=40$ the beam spreads-out, the spatial localisation disappears and the wave takes effectively form of a spherical wave. In fact in Fig. 8 is depicted the first part of the propagation path of the Fig. 9 .

The reflected wave is calculated assuming the critical angle $\theta_{c}=10^{\circ}$ and the angle of beam incidence $\theta_{d 0}=50^{\circ}$. Propagation of the reflected beam is depicted in Fig. 10 showing its slightly different propagation characteristics as compared to the incident wave in Fig. 8.

Table 1. The angles (in degrees) $\theta_{q n}$ pertaining to $\mathrm{A}: \lambda / \lambda_{q \min }=$ 0.2 and $\mathrm{B}: \lambda / \lambda_{q \min }=0.36$

\begin{tabular}{cccccccc}
\hline & $n=1$ & $n=2$ & $n=3$ & $n=4$ & $n=5$ & $n=6$ & $n=7$ \\
\hline $\mathrm{A}$ & 2.9 & 5.7 & 8.6 & 11.5 & 14.5 & 14.5 & 20.5 \\
$\mathrm{~B}$ & 5.2 & 10.4 & 15.7 & 21.1 & 26.7 & 32.7 & 39.1 \\
\hline
\end{tabular}

Table 2. The angles (in degrees) $\Psi_{0}, \Psi_{0 n}$ and $\Psi_{q n}$

\begin{tabular}{lcccccccc}
\hline & & $n=1$ & $n=2$ & $n=3$ & $n=4$ & $n=5$ & $n=6$ & $n=7$ \\
\hline A & 98.5 & 98.5 & 98.5 & 98.4 & 98.4 & 98.3 & 98.2 & 81.9 \\
A & $\Psi_{q n}$ & 5.9 & 11.8 & 17.7 & 23.7 & 29.8 & 36.0 & 42.3 \\
B & 98.5 & 98.5 & 98.4 & 98.3 & 98.1 & 97.6 & 96.8 & 86.5 \\
B & $\Psi_{q n}$ & 10.6 & 21.3 & 32.2 & 43.5 & 55.4 & 68.4 & 84.6 \\
\hline
\end{tabular}

The zoomed central part of the initial profiles for $\zeta=$ $0, \eta=0$, of the incident and reflected beam waves are 
in Fig. 11, showing the Goos-Hänchen shift (the curves a and $b$ ). The curve $c$ shows larger shift of the (1.8 times) narrower beam with respect to wavelength.

To the $N=4$ spatial spectral plane wave components pertain the propagation angles shown in Table 1, the case $\mathrm{A}$ for the broader beam and the case $\mathrm{B}$ for the narrower beam with respect to the wavelength. The pertaining angles of the reflection factors $\Psi_{0}, \Psi_{0 n}$ and $\Psi_{q n}$ are shown in Table 2.

If one takes instead of $N=4$, ie instead of 9 sampling points, higher number of harmonics $N=7$, ie 15 sampling points, one arrives at the Goos-Hänchen shift depicted in Fig. 12. This is with $\theta_{q 7}=39.1^{\circ}$ and $\Psi_{q 7}=$ $84.6^{\circ}$ near to the limiting case $\theta_{q \max }=40^{\circ}, \Psi_{q \max }=90^{\circ}$ for $\theta_{c}=10^{\circ}$ and $\theta_{d 0}=50^{\circ}$.

\section{Conclusions}

The reflection and refraction of beam waves on the planar dielectric dielectric boundary has been thoroughly analysed. The simple beam formation in terms of Fourier series of discrete homogeneous plane wave components has been elaborated.

The analysis shows that the more spectral components one takes, the narrower the beam is. But the GoosHänchen shift never exceeds $90^{\circ}$, ie one quarter of the period of the highest harmonics - in our case approximately one quarter of the beam width. Therefore making the beam narrower cannot increase the relative beam shift magnitude.

As shown, there is a fundamental difference between the homogeneous and non-homogeneous plane wave. In case of the homogeneous plane wave, the power flows along the boundary plane only, and no power penetration through the boundary plane exists. For an nonhomogeneous plane wave a steady flow of the power density through the boundary between the two media exists transporting the power from incident to the reflected wave through the second media and so giving the rise to the shifted wave.

\section{Acknowledgements}

This work has been supported by the Slovak Research and Development Agency under the contract no. APVV16-0059 and by the Scientific Grant Agency of the Ministry of Education, Science, Research and Sport of the Slovak Republic and in part by the Slovak Academy of Sciences, under project no. 1/0405/16.

\section{REFERENCES}

[1] F. Goos and H. Hänchen, "Ein neuer und fundamentaler Versuch zur Totalreflexion", Ann. Phys., vol. 436 (1947), pp. 333-346.
[2] F. I. Fedorov, "To the theory of total reflection, (K teorii polnogo otraženija)", Dokl. Akad. Nauk SSSR, vol. 105 (1955), pp. 465468 in Russian.

[3] B. R. Horowitz and T. Tamir, "Lateral Displacement of a Light Beam at a Dielectric Interface", J. Am. Opt. Soc., .vol. 61, (1971), no. 5, pp. 586-594.

[4] A. W. Snyder and J. D. Love, "Goos-Hanchen shift", Appl. Opt., vol. 15 (1976), no. 1, pp. 236-238.

[5] V. G. Fedoseyev, "Energy motion on total internal reflection of an electromagnetic wave packet", J. Opt. Soc. Am., vol. A3 (1986), pp. 826-829.

[6] S. R. Seshadri, "Goos-Hanchen beam shift at total internal reflection", J. Opt. Soc. Am., vol. A5 (1988), no. 4, pp. 583-585.

[7] L'. Šumichrast and J. Franek, "Numerical treatment of the spatio-temporal electromagnetic beam-wave packet" Journal of Electrical Engineering, vol. 68 (2017), No. 2, pp. 109-116.

[8] A. Papoulis; The Fourier Integral and Its Applications, McGraw Hill, New York 1962.

Received 14 November 2017

Lubomír Šumichrast (Doc, Ing, PhD) is an emeritus Associate Professor with the Faculty of Electrical Engineering and Information Technology of the Slovak University of Technology. His main research interests include the electromagnetic waves propagation in various media and structures, computer modelling of wave propagation effects as well as optical communication and integrated optics.

Jaroslav Franek (Ing, CSc), graduated from the Faculty of Electrical Engineering, Slovak Technical University, Bratislava, in 1969, and received the $\mathrm{CSc}(\mathrm{PhD})$ degree in Physics in 1986. At present he is an emeritus Assistant Professor at the same Faculty. The main fields of his research are circuit and electromagnetic field theory, namely the microwave technology.

Rastislav Dosoudil (Doc, Ing, PhD) was born in Bratislava, in 1970. He graduated from Faculty of Electrical Engineering and Information Technology, Slovak University of Technology, in Bratislava, in Material Engineering branch, 1993 (technology of electronic equipments) and received the PhD degree in Theory of Electromagnetism in 2000. At present, he is an Associate Professor (2008) "with the Institute of Electrical Engineering. He teaches subjects concerning electric circuits, electromagnetics and modern materials for electrical engineering. His research activities are mainly electromagnetic composite materials, high-frequency complex permittivity/permeability phenomena, electromagnetic wave absorbing properties of composites, and magnetic measurements.

Jozefa Červeňová (Doc, Ing, $\mathrm{PhD}$ ), born in Bratislava, graduated from the Faculty of Electrical Engineering and Information Technology of the Slovak University of Technology in Bratislava in 1971. She completed her PhD studies in 1993 in the field of Electromagnetic Theory. Her current research interests concern mainly the testing methods of optical fiber systems and components. 